\title{
Flower Image Retrieval Using LBP, Wavelet Moments, Gabor Wavelet Features and Multiple Distance Functions
}

\author{
Zannatul Azme, Khadiza Sultana Happy, Mohammad Farhad Bulbul* \\ Department of Mathematics, \\ Jessore University of Science and Technology, Jessore, Bangladesh \\ farhad@just.edu.bd
}

\begin{abstract}
Flower image retrieval is a significant and challenging problem in content-based image retrieval. In this paper, we propose a content-based method for retrieving flower images of specified specie from a database of flower images of various species. Firstly, we use wavelet moment, Gabor wavelet and Local Binary Pattern (LBP) independently to characterize all flower images in the database. Secondly, we represent a query flower image with Gabor wavelet, wavelet moment, and LBP individually and search images in the database analogous to the query image. The retrieval is accomplished through calculating similarities between the query image and the database images by employing a set of distance functions. Experimental evaluation of the approach reveals that the Gabor wavelet achieves superiority over the wavelet moment and LBP considerably. It is also indicated that the retrieval outcome can be improved through concatenating the Gabor wavelet, wavelet moment, and LBP features rather than utilizing them individually.
\end{abstract}

\section{Introduction}

At present world, with the availability of image capturing devices such as digital cameras, mobile cameras, webcams, video cameras, image scanners, the size of digital image collection is increasing at a great rate. A computer system for image searching, browsing and retrieving from a large database of digital images is called an image retrieval system. It is a tool, required by users from various domains, including remote sensing, medicine, crime prevention, architecture, publishing, fashion, etc. From this point of view, there have been developed many prevalent image retrieval systems. There are two frameworks of image retrieval such as text-based image retrieval and content-based Image Retrieval. The text-based approach started since the early 1960s. In text-based image retrieval system, for finding similar types of images it is needed to search by the accurate textual description, filenames, caption, keywords etc. Because the images are annotated in the database by textual description, filenames, caption, keywords etc. But text- based image retrieval is oldest image searching system. There are many several problems of text- based image retrieval methods. Firstly, annotation is never complete because it depends on the goal of the annotation, some mistakes like spelling error, spelling difference (US vs.UK), weird abbreviation (particularly medical) etc. The use of keywords is not only complex but also insufficient to represent the content by the size of image databases growing etc. The keywords are not unique for one kind of searching. The problems of image queries by text-based approaches cannot be described at all, but tap into the visual features of images .For example, a query for all the images in the database with "classroom" in it will good results if we annotate all the images containing classroom but for the same annotations, a specific search for images with a chair, table, computer or student in tit will fail. Another problem with text based approach is the lack of uniform 
Zannatul Azme, Khadiza Sultana Happy, Mohammad Farhad Bulbul; Flower Image Retrieval Using LBP, Wavelet Moments, Gabor Wavelet Features and Multiple Distance Functions. Advances in Image and Video Processing, Volume 6 No 5, October (2018); pp: 1-12

textual descriptions of such attributes like color, shape, texture etc. So users are not satisfied with the conventional text-based retrieval methods. CBIR that means Content-based Image Retrieval is a technique that helps to access and arrange the digital images from a giant database by using visual contents of the images i.e. the features of images. The word "Content- based" express that the query will be analyzed by the actual contents of the image other than the metadata such as tags, keywords or descriptions linked with the image. Here the 'content' means colors and textures information that can be extracted from the image. The focus of CBIR is to avoid the use of textual descriptions. CBIR is such a process that will ease the data handling and the user can easily access the data. Basically, the technique tries to retrieve images similar to a user-defined specification or pattern (e.g., shape sketch, image example). So in CBIR, retrieving of an image based on focusing at their contents like colors, textures, shapes etc. The CBIR process has gain utmost importance because most web-based image search engines purely depend on metadata and this produces a lot of false identification in the search results. For these issues, CBIR has become an active and fast moving research sector since the 1990s. There are several advantages of CBIR in real time situation. Two major research communities, database management and computer vision have become a very active area of research on CBIR. CBIR technique is easy, effective, and less expensive. It takes less time to find all those related images. All over that, present world image retrieval mainly focused on Content-based image retrieval system. The possibility of an automatic retrieval process is one of the main advantages of the CBIR approach, instead of the regular keyword-based approach, which usually time-consuming and very laborious. However, CBIR technology has been used in several applications such as fingerprint identification, biodiversity information system678s, digital libraries, Crime prevention, Security Check, Medical Diagnosis, Intellectual Property, Art collection, Medical Image Databases, Scientific Databases, General Image collections for licensing, Architectural and Engineering design, Fashion and publishing, Military, photograph archives, face finding, geographical information and remote sensing systems and so on.

Flower image retrieval system is one type content-based image retrieval system and it is very significant for computer-aided plant species recognition. Flower image databases and collections can be enormous in size, containing hundreds, thousands or even millions of flower images. Difficulties for flower image retrieval are as follows there complex backgrounds, variations of brightness and intensity under different natural illuminations, shadow effects of the surrounding, diversity of flowers as different appearances, different camera angles, different sizes, and different resolution, etc. As a result, flower image retrieval is a significant and challenging problem in content-based image retrieval. We had systematic and overall researches on flower images, feature fusion, regional segmentation and feature extractions etc. [1], [2]. There are about 250000 named kinds of flowering plants. But many plant species that have not been classified and named. Plant organization and identification is a very ancient area. A major development can be projected if the plant identification can be carried out by a computer automatically with the aid of image processing and computer vision techniques, and many data management techniques.

Many researchers have been worked at flower image retrieval system. For example, in 2008, MariaElena Nilsback and Andrew Zisserman [2] introduced a 103 class flower dataset. They compute four different features for the flowers, each describing different aspects, namely the local shape/texture, the shape of the boundary, the overall spatial distribution of petals, and the color. They combined the features using a multiple kernel framework with an SVM classifier. The weights for each class are learnt using the method of Varma and Ray [3], which has achieved state of the art performance on other large dataset, such as Caltech 101/256. Their dataset had a similar challenge in the number of classes, 
but with the added difficulty of large between class similarity and small within class similarity. Their results showed that learning the optimum kernel combination of multiple features vastly improves the performance, from $55.1 \%$ for the best single feature to $72.8 \%$ for the combination of all features. After two years, Xiao Ke et al. [4] discussed on recent developments in content-based flower image retrieval. They studied repetitive image detection algorithm based on canny edge to filter repetitive images in flower database and adaptive threshold segmentation algorithm based on 2RGB mixed color to segment flower image. They showed this segmentation results of these flowers are not sampling that is these flowers was not completely extracted from entire images. Again, they tested adaptive threshold segmentation algorithm based on $2 \mathrm{RGB}$ mixed-color model used in these part of testing. These types of tested flowers were similar to other kinds of flowers. Finally to test the flower image retrieval system, they used HSV histogram, color moments, GLCM, Gabor texture, weighted invariant moments and edge LBP features. They also tested the retrieval results by using the strategy of multifeature fusions. They obtained very good retrieval results of flower image retrieval system based on multi-feature fusions. They used total 15 categories flower images, under the premise of returning 20 most similar images for each test image, the obviousness of 12 categories' flowers passed $80 \%$ and other three kinds of flowers passed $70 \%$.

In the same year, Hsu et al. [5] presented an interactive system for recognizing flower images used by digital cameras. They proposed a system which provides a simple user interface which allows each user to draw a rectangular bounding window containing the interested flower region. They developed to find the flower boundary as accurately as possible by a boundary tracing algorithm. Along the color and shape features of the total flower region, the color and shape features of the pistil/stamen area are also removed to represent the flower characteristics in a more appropriate currency. They showed their experiments conducted on two different flower image databases consisting of 24 species and 102 species. Finally, they proposed system outperforms other approaches in terms of the recognition rate than the methods proposed by Hong et al. [6], Zou and Nagy [7], and Saitoh et al. [8].

Several years later, HU et al. [9] developed another content-based flower image retrieval system. They researched on flower image retrieval algorithm based on saliency map. They avoided traditional image segmentation. Firstly, they improved and used Itti's visual attention model, and extracted the color and LBP texture features using the saliency map. They gained more effective retrieval results than the other results based on the saliency map extracted through Itti's model.

In this paper, we propose a system for content-based flower image retrieval. For a given query flower image, our system goals to return a set of representative images in which similar types of flowers look like. We use some descriptors such as wavelet moments, Gabor wavelet and LBP to represent the query image as well as the database images. A set of distance functions is utilized to measure image similarity. In this work, we build the system by using the Gabor wavelet, wavelet moments, and LBP descriptors individually and collectively.

The rest of this paper is organized as follows. The proposed approach is presented in Section 2. The experimental results are demonstrated in Section 3. Finally, Section 4 contains a brief conclusion of this work.

\section{Our approach}

In this section, we discuss about our approach for retrieving flower images. We firstly discuss on the feature extraction from query and database flower images and then about the distance functions used for finding out the similarity between the query image and the database images.

Figure 1 represents our architectural overview of the proposed framework. 


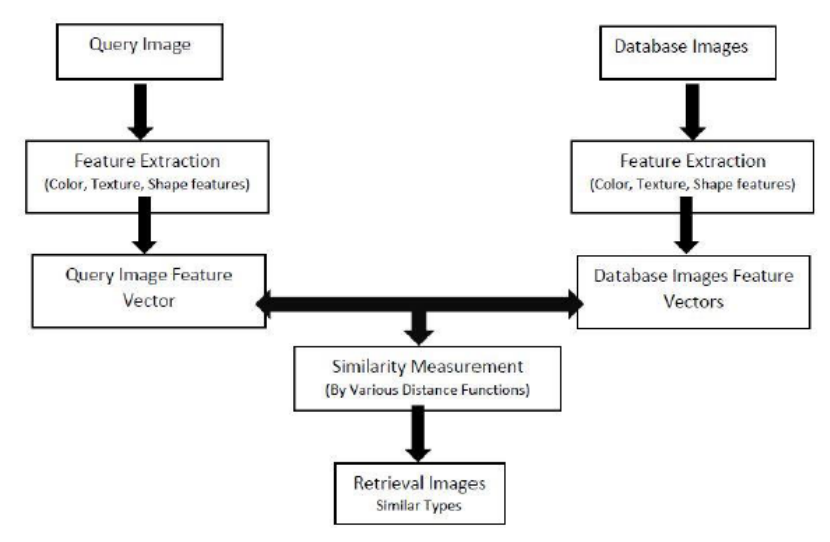

Figure 1: Architectural overview of the proposed framework

\subsection{Feature Extraction}

Our work uses wavelet moment, Gabor wavelet and Local Binary Pattern (LBP) features to describe a flower image. This section discusses those features briefly.

Wavelet Transform: A wavelet is a small wave. Wavelet transform is the representation of a function by wavelets. It is used for extraction of texture feature. Wavelets are defined as small waves of different frequency having limited [10]. Wavelet transform is broadly used in computer vision as an image processing technique for object identification and classification. Application of Discrete Wavelet Transform (DWT) as an image processing system produces the revolution values called wavelet coefficient. A common method of feature extraction from wavelet transformation is the calculation of coefficient distribution over elected mother of wavelet. Naturally, the actualization of DWT uses the mother of wavelet such as Haar, daubechies, Coiflet, Meyer, Morlet and Mexican Hat. However, this study only considers the Coiflet mother wavelet. The fundamental idea behind wavelets is to analyze signal according to scale. The basic idea of DWT is to provide the time-frequency representation. The 2D-DWT represents an image in terms of a set of shifted and dilated wavelet functions $\psi L C, \psi C L, \psi C C$ and scaling function $\phi L L$ that form an orthonormal basis for $L 2(R 2)$. Given J-scale DWT as image $x(s, t)$ of $\mathrm{N} \times \mathrm{N}$ is decomposed as

$$
\begin{gathered}
x(s, t)=\sum_{k, i=0}^{N_{J^{-1}}} U_{J, k, i} \phi_{J, k, i}^{L L}(s, t)+\sum_{\beta \in B} \sum_{j=1} \sum_{k, i=0}^{N-1} W_{j, k, i}^{B} \psi_{j, k, i}^{B}(s, t) \\
\text { Where, } \begin{aligned}
\phi_{j, k, i}^{L L}(s, t) & \equiv 2^{-j / 2} \phi\left(2^{-j} s-k, 2^{-j} t-i\right), \psi_{j, k, i}^{B}(s, t), \psi_{j, k, i}^{B}(s, t) \\
& \equiv 2^{-j / 2} \psi^{B}\left(2^{-j} s-k, 2^{-j} t-i\right), B \in \boldsymbol{B}, \boldsymbol{B}
\end{aligned}
\end{gathered}
$$

$\{\mathrm{LC}, \mathrm{CL}, \mathrm{CC}\}$ and $N j=N 2 j /$. In this paper $\mathrm{LC}, \mathrm{CL}$, and $\mathrm{CC}$ are called wavelet or DWT sub-bands. $U_{j, k, i}=\iint x(s, t) \phi J, k, i d s d t$ is a scaling coefficient and $W_{j, k, i B}=\iint x(s, t) \psi_{j, k, i B} d s d t$ denotes the $(\mathrm{k}, \mathrm{i})$ th wavelet coefficient in scale $j$ and sun-band $B$ [11]. Figure 2 represents the scaling model in wavelet transform. 


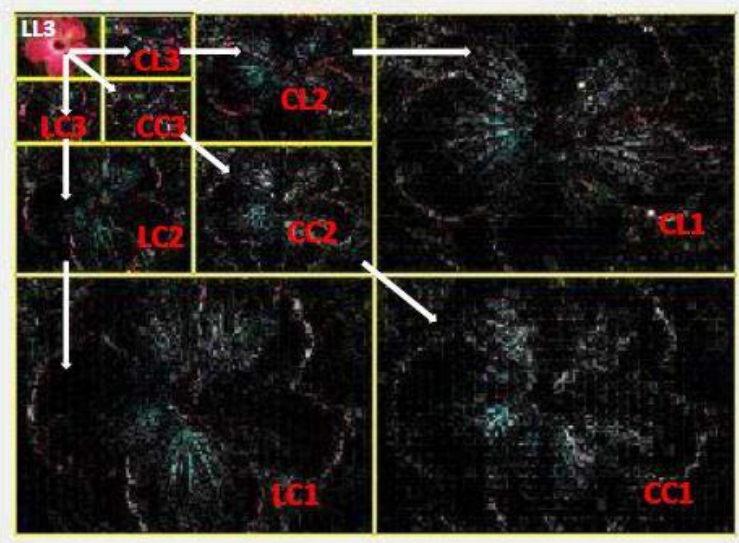

Figure 2: Joint spatial and frequency representation of a 2-D three-scale DWT

We use the image processing method that have been applied on the feature vector extraction. Applying the wavelet transform to the image with a 3-level decomposition, the standard deviation and mean of the transform coefficient $\mathrm{CC} 1$ is used to form the feature vector.

Gabor Wavelet: Let an image be I $(u, v)$ with $m \times n$ size. Then its discrete Gabor wavelet transform is given by a convolution [12]:

$$
G_{p q}(u, v)=\sum_{x} \sum_{y} I(u-x, v-y) \psi_{p q}^{*}(x, y)
$$

where,

$x$ and $y$ are the filter mask size variables

$\psi p q *$ is the complex conjugate of $\psi p q$.

$\psi p q$ is a class of self-similar functions generated from dilation and rotation of the following mother wavelet:

$$
\psi(s, t)=\frac{1}{2 \pi \sigma_{s} \sigma_{t}} \exp \left[-\frac{1}{2}\left(\frac{s^{2}}{\sigma_{s}^{2}}+\frac{t^{2}}{\sigma_{t}^{2}}\right)\right] \cdot \exp (j 2 \pi V s)
$$

Here,

$V=$ modulation frequency.

The self-similar Gabor wavelets are obtained through the generating function:

$$
\psi_{p q}(s, t)=a^{-p} \psi(\tilde{s}, \tilde{t})
$$

Here,

$p, q$ respectively denotes the scale and orientation of the wavelet with $p=0,1, \ldots P-1, q=0,1, \ldots, Q-1$

And

$$
\begin{aligned}
& \tilde{s}=a^{-p}(s \cos \theta+t \sin \theta) \\
& \tilde{t}=a^{-p}(-s \sin \theta+t \cos \theta) \quad, a>1 \text { and } \theta=\frac{q \pi}{Q}
\end{aligned}
$$


Zannatul Azme, Khadiza Sultana Happy, Mohammad Farhad Bulbul; Flower Image Retrieval Using LBP, Wavelet Moments, Gabor Wavelet Features and Multiple Distance Functions. Advances in Image and Video Processing, Volume 6 No 5, October (2018); pp: 1-12

Local binary patterns (LBP): Local binary pattern (LBP) is a type of visual descriptor used for pattern classification in the area of computer vision [13]. LBP features encode local texture information, which we can use for tasks such as classification, detection, and recognition. LBP is a simple yet very efficient texture operator. The LBP operator typically works in $3 \times 3$ pixel blocks. All working block are thresholded by the center pixel. Its weighted by powers of 2 and then mixed to label the center pixel. These labels are the pixels of a picture by thresholding the neighborhood of every pixel then the result as a binary number are conceive. The operator can also be extended to neighborhood with various sizes [14]. Let us consider a neighborhood denoted by $(P, Q)$, here $P$ represents the sampling points and $Q$ represents the radius. These sampling points lie around the center pixel $(U, V)$ and at coordinates are

$$
\left(U_{m}, V_{m}\right)=(U+Q \cos (2 \pi m / P), V-Q \sin (2 \pi m / P))
$$

Now the LBP label for pixel $(U, V)$ can be calculated as follows

$$
L B P_{P, Q}(U, V)=\sum_{m=1}^{P-1} T\left(f\left(U_{m}, V_{m}\right)-f(U, V)\right) \cdot 2^{m-1}
$$

Where $T(U)=1$ if $(U \geq 0)$ and $T(U)=0$ if $U<0[15]$.

Accordingly pixel value is summed for the LBP number of this texture unit. LBP technique is gray scale invariant and can be easily joined with a simple contrast amount by adding for each neighborhood the difference of the average gray level of those pixels which have the value 1 and those which have the value 0 respectively[16].

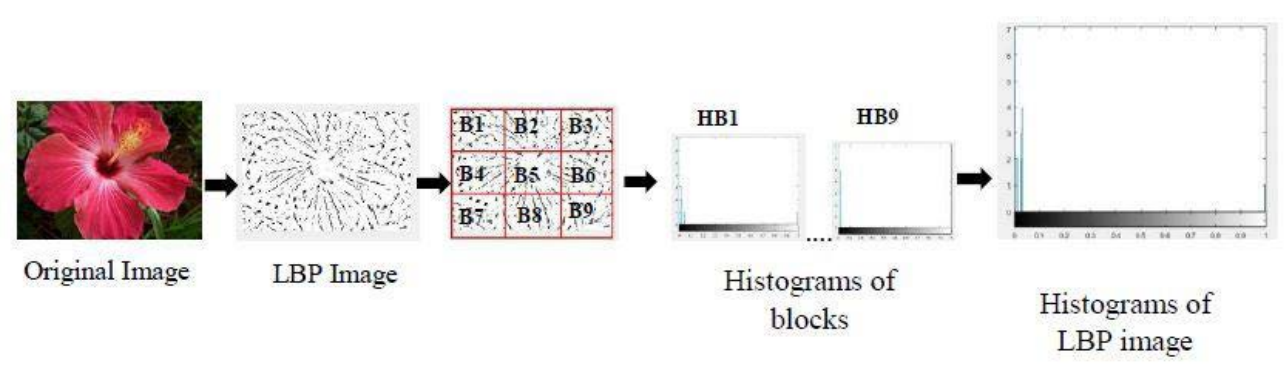

Figure 2: LBP coded image of a flower image

\subsection{Distance Function}

The concept of distance function has been developed to provide a formal description for measuring distance between two points in a vector space. In the section, we discuss about six distance function such as L2, chebyshev, cosine, correlation, city block and minkowski. These functions are used in our work to measure similarities between query image and the database images.

L2 Distance Function: The L2 distance between two points $a$ and $b$ is the length of the line segment between them.

If $a=\left(a_{1}, a 2, \ldots a m\right)$ and $b=(b 1, b 2, \ldots b m)$ are two points in Euclidian $\mathrm{n}$-space then the distance $\mathrm{d}$ between $a$ and $b$ is given by

$$
d(a, b)=\sqrt{(a 1-b 1) 2}+(a 2-b 2) 2+\cdots+(a m-b m) 2
$$




$$
=\sqrt{\sum_{j=1}^{m}\left(a_{j}-b_{j}\right)^{2}}
$$

where $\mathrm{a}$ and $\mathrm{b}$ is represented as Euclidian vectors which are starting from the origin of the space with their terminal points and ending at the two points.

$$
\begin{aligned}
& \text { Euclidean norm of a is, }\|a\|=\sqrt{a \cdot a}=\sqrt{a_{1}{ }^{2}+a_{2}{ }^{2}+\ldots+a_{m}{ }^{2}} \\
& \text { And } \quad \mathrm{b} \text { is, }\|b\|=\sqrt{b \cdot b}=\sqrt{{b_{1}{ }^{2}+b_{2}{ }^{2}+\ldots+b_{m}{ }^{2}}^{2}}
\end{aligned}
$$

The $\mathrm{L} 2$ distance between $\mathrm{a}$ and $\mathrm{b}$ is

$$
\begin{aligned}
\|b-a\| & =\sqrt{(b-a) \cdot(b-a)} \\
& =\sqrt{\|a\|^{2}+\|b\|^{2}-2 a \cdot b}
\end{aligned}
$$

Chebyshev Distance Function: This distance function examines the entire magnitude of the difference between coordinates of a couple of objects. We use this function for measuring the similarity between the query flower image and the flower images into the data set.

$$
\text { The formula is, } d_{s}=\left|x_{m k}-x_{n k}\right| I
$$

In Chebyshev distance, all 8 neighboring cells from the given point can be reached by one unit [17].

Cosine Distance function: We use Cosine Similarity for finding the similarity between the query flower image and the dataset flower images. It finds similarity between two nonzero vectors of an inner product space. It measures the cosine of the angle between the vectors.

The cosine of two non-zero vectors is derived from the Euclidean dot product formula [18] ie. $P . Q=\|P\|\|Q\| \cos \theta(16)$

Then the similarity is measured as,

$$
\begin{gathered}
\text { Similarity, } \quad \cos \theta=\frac{P . Q}{\|P\|\|Q\|} \\
=\frac{\sum_{i=1}^{n} P_{i} Q_{i}}{\sqrt{\sum_{i=1}^{n} P_{i}^{2}} \sqrt{\sum_{i=1}^{n} Q_{i}{ }^{2}}}
\end{gathered}
$$

Where, $P i Q i$ are the components of $P Q$ vectors.

Correlation: Correlation measures similarity rather than dissimilarity or distance. It is standardized angular separation by centering the coordinates to its mean value.Correlation distance function calculates both linear and nonlinear association between two or random vectors. Firstly, it calculates the distance correlation between two random vectors. Then, compare this value to the distance correlations of many shuffles of the data.

The formula is [19], 


$$
d_{s}=\frac{\sum_{i=1}^{n}\left(x_{p i}-\overline{x_{p}}\right) \cdot\left(x_{q i}-\overline{x_{q}}\right)}{\left(\sum_{i=1}^{n}\left(x_{p i}-\overline{x_{p}}\right)^{2} \cdot \sum_{i=1}^{n}\left(x_{q i}-\overline{x_{q}}\right)^{2}\right)^{\frac{1}{2}}}
$$

City block distance function: The City block distance is explained as if we consider two points in the $x y$-plane, the City block distance is instead measured as the distance in $\mathrm{x}$ plus and the distance in $\mathrm{y}$. This is similar to the way if we move in a city, instead of going straight through we have to move around the buildings.

The City block distance between two points, $p$ and $q$, with the dimensions $\mathrm{n}$ is determined as [20]:

$$
\text { City block distance }, \quad C D=\sum_{i=1}^{n}\left|p_{i}-q_{i}\right|, \quad C D \geq 0
$$

\section{$C D=0$, for identical points $C D>0$, for Showing little similarity points}

The given figure displays an example of two points namely $p$ and $q$. There are five values for each point i.e. $i=5$. The dotted lines are the distances $\left(p_{1}-q_{1}\right),\left(p_{2}-q_{2}\right),\left(p_{3}-q_{3}\right),\left(p_{4}-q_{4}\right)$ and $\left(p_{5}-q_{5}\right)$ which are go into in the above equation.

Results produced by city block distance are similar to the Euclidean distance. With this distance function in a single dimension, the effect of a large difference is reduced as the distances are not squared.

Minkowski Distance Function: The Minkowski distance is a metric in a normed vector space which can be considered as a generalization of both the Euclidean distance and the Manhattan distance.

The Minkowski distance of order $m$ between two points [21]

$$
\begin{gathered}
U=\left(u_{1}, u_{2}, \ldots, u_{n}\right) \text { and } V=\left(v_{1}, v_{2} \ldots v_{n}\right) \in \mathbb{R}^{n} \\
D(U, V)=\left(\sum\left|u_{i}-v_{i}\right|^{m}\right)^{\frac{1}{m}}
\end{gathered}
$$

When, $m<1$, the distance between $(0,0)$ and $(1,1)$ is $2^{1 / m}>2$

\section{Experimental Results and Discussion}

This section firstly discusses the image dataset construction and then the experiments to evaluate the proposed method using the dataset are carried out.

\subsection{Image Dataset}

To make flower image database, we download the pictures of flowers from Google. The dataset consists of 500 flower images of 20 species and there are 25 flower images of each specie. It is a challenging dataset as some flowers have similar types of texture description but those flowers are not of the same categories. Thus, it is difficult to separate the flower images. We actually arrange our flower dataset in this way so that this type of problems can arise and we try to introduce a method to solve those problems. Figure 2 represents a chunk of our dataset of different flower images. 


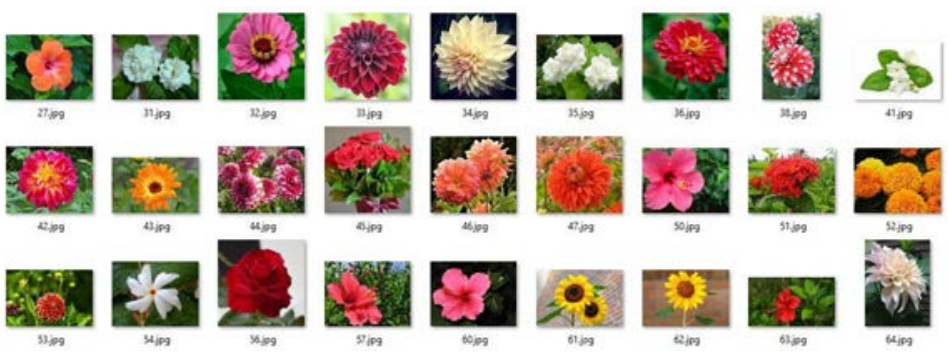

Figure 2: Example flower images of dataset

\subsection{Retrieval Results on Individual Feature}

We calculate our results with a target for retrieving 20 similar images from our constructed dataset. For each feature and a single distance function, we observe the retrieval outcomes of our system on 5 types of flowers at least.Then, we take the average of retrieval accuracies corresponding to 5 types of flowers. The retrieval results for wavelet moments, Gabor wavelet and LBP feature and for several distance functions are shown in Table 1, Table 2, and Table 3 respectively. Indeed, we calculate retrieval accuracy for using wavelet moments, Gabor wavelet and LBP on the same set of distance functions.

In Table 1, we observe that the maximum result for the wavelet moments is $20 \%$ for Chebychev distance function (bold-faced). Also, we observe that the maximum result for the Gabor wavelet is $20 \%$ for city block and Chebychev distance function in Table 2 . The maximum result for the LBP is $15 \%$ for city block distance function in Table 3. Note that there is a variation in retrieval results for the different distance functions in different features. However, a comparison among retrieval rates for wavelet moments, Gabor wavelet and LBP is represented in Figure 3. It is observable that the result for LBP feature is inferior compared to wavelet moments and Gabor wavelet. The maximum result for LBP feature is $15 \%$ for city block distance function and the correlation distance function. The city block and the cosine distance function are better for Gabor wavelet feature than the other distance functions. Using this distance function, we get $20 \%$ and $15 \%$ accurate result respectively for Gabor wavelet feature. But the Chebychev distance function is better for wavelet Moments. For this function, wavelet moments feature provides $20 \%$ accurate result

Table 1: Retrieval accuracy using wavelet moments feature

\begin{tabular}{|l|l|l|l|}
\hline Distance function & Retrieval target & No. of retrieve image & Average Accuracy (\%) \\
\hline L2 & 20 & 2 & 10 \\
\hline City block & 20 & 3 & 15 \\
\hline Minkowski & 20 & 3 & 15 \\
\hline Chebychev & $\mathbf{2 0}$ & $\mathbf{4}$ & $\mathbf{2 0}$ \\
\hline Cosine & 20 & 2 & 10 \\
\hline Correlation & 20 & 3 & 15 \\
\hline
\end{tabular}

Table 2: Retrieval accuracy using Gabor wavelet feature

\begin{tabular}{|l|l|l|l|}
\hline Distance function & Retrieval target & No. of retrieve images & Average Accuracy (\%) \\
\hline L2 & 20 & 2 & 10 \\
\hline City block & $\mathbf{2 0}$ & $\mathbf{4}$ & $\mathbf{2 0}$ \\
\hline Minkowski & 20 & 2 & 10 \\
\hline
\end{tabular}

\begin{tabular}{|l|l|l|l|}
\hline Chebychev & 20 & 2 & 10 \\
\hline Cosine & $\mathbf{2 0}$ & $\mathbf{4}$ & $\mathbf{2 0}$ \\
\hline Correlation & 20 & 3 & 15 \\
\hline
\end{tabular}


Table 3: Retrieval accuracy using LBP feature

\begin{tabular}{|l|l|l|l|}
\hline Distance function & Retrieval target & No. of retrieve image & Average Accuracy (\%) \\
\hline L2 & 20 & 2 & 10 \\
\hline City block & $\mathbf{2 0}$ & $\mathbf{3}$ & $\mathbf{1 5}$ \\
\hline Minkowski & 20 & 2 & 10 \\
\hline Chebychev & 20 & 2 & 10 \\
\hline Cosine & 20 & 1 & 5 \\
\hline Correlation & 20 & 3 & 15 \\
\hline
\end{tabular}

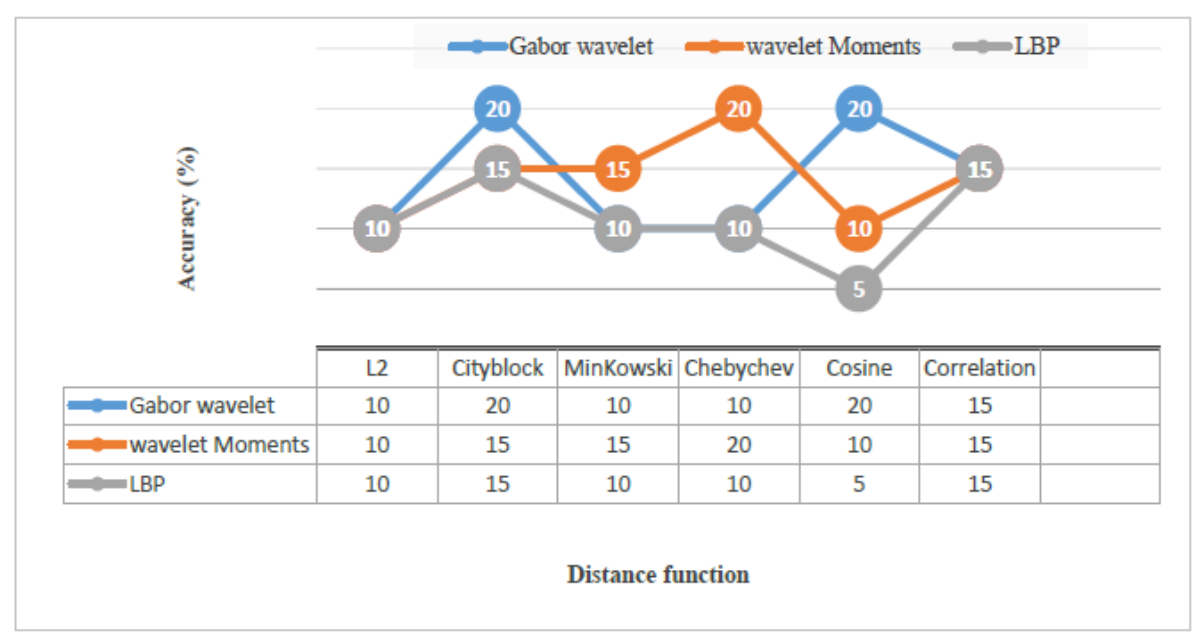

Figure 3: Comparison of retrieval rates for wavelet moments, Gabor wavelet and LBP, features

\subsection{Retrieval Results on Combined Feature}

To improve the retrieval outcome, the combination of wavelet moments, Gabor wavelet, and Local Binary Pattern (LBP) is considered. Table 4 shows the retrieval result on the concatenation of those features. The highest retrieval result of $35 \%$ is achieved when the cosine distance function is employed on the output of the integration of wavelet moments, Gabor wavelet, and Local Binary Pattern (LBP) feature vectors. It is worth to mention that the proposed system improves the retrieval accuracy by $15 \%$ when the wavelet moments, Gabor wavelet, and Local Binary Pattern (LBP) are combined rather than using them alone.

Table 4: Retrieval accuracy on the combination of wavelet moments, Gabor wavelet, and LBP

\begin{tabular}{|l|l|l|l|}
\hline $\begin{array}{l}\text { Distance } \\
\text { Function }\end{array}$ & Retrieval Target & No. of Retrieve Image & Average Accuracy (\%) \\
\hline L2 & 20 & 4 & 20 \\
\hline City block & 20 & 5 & 25 \\
\hline Minkowski & 20 & 5 & 25 \\
\hline Chebychev & 20 & 6 & 30 \\
\hline Cosine & $\mathbf{2 0}$ & 7 & $\mathbf{3 5}$ \\
\hline Correlation & 20 & 6 & 30 \\
\hline
\end{tabular}

\section{Conclusion}

In this paper, we have calculated separately the retrieval accuracy for retrieving similar type of flower images for three descriptors such as Wavelet moments, Gabor wavelet and LBP. We observe that the result for wavelet moments and LBP feature are inferior compared to Gabor wavelet. We have also calculated retrieval outcomes for combining Wavelet moments, Gabor wavelet and LBP. The retrieval 
results gain the improvement for the combination of those features and better than the situation when the features are utilized alone. Furthermore, there is a variation in results for the different distance functions with the features. Some distance functions are giving higher results for some features only. The City block and correlation functions are good for all the features, whereas the minkowski and the chebychev function is good for only some specific features instead of all.

\section{REFERENCES}

[1] Nilsback M, Zisseman A. "A Visual Vocabulary for Flower Classification," Institute of Electrical and Electronics Engineers (IEEE) Computer Society Conference on Computer Vision and Pattern Recognition, vol.2, pp.1447-1454. 2006..

[2] Nilsback M, Zisseman A. “Automated Flower Classification over a Large Number of Classes," Sixth Indian Conference on Computer Vision,Graphics\&Image Processing,pp.722-729, Dec.2008.

[3] M. Varma and D. Ray. Learning the discriminative power invariance trade-off. In Proc. International Conference on Computer Vision(ICCV), 2007.

[4] Ke, Xiao, Shaozi Li, and Xiaofen Chen. "Modified model in content-based flower image retrieval." Intelligent Computing and Intelligent Systems (ICIS), 2010 Institute of Electrical and Electronics Engineers (IEEE) International Conference on. Vol. 3. IEEE, 2010.

[5] [5] Hsu, Tzu-Hsiang, Chang-Hsing Lee, and Ling-Hwei Chen. "An interactive flower image recognition system." Multimedia Tools and Applications 53.1 (2011): 53-73.

[6] Hong A, Chen G, Li J, Chi Z, Zhang D (2004) A flower image retrieval method based on ROI feature. Journal of Zhejiang University (Science) 5(7):764-772

[7] Zou, Jie, and George Nagy. "Evaluation of model-based interactive flower recognition." null. Institute of Electrical and Electronics Engineers (IEEE), 2004

[8] Saitoh, Takeshi, Kimiya Aoki, and Toyohisa Kaneko. "Automatic recognition of blooming flowers." null. Institute of Electrical and Electronics Engineers (IEEE), 2004.

[9] Hu, Xuelong, et al. "Flower image retrieval based on saliency map." Computer, Consumer and Control (IS3C), 2014 International Symposium on. Institute of Electrical and Electronics Engineers (IEEE), 2014.

[10] Rafael C. Gonzalez, and E. Richard, "Digital Image Processing," Prentice Hall Press, Second Edition, 2002.

[11] Ghazali, Kamarul Hawari, et al. "Feature extraction technique using discrete wavelet transform for image classification." Research and Development, 2007. SCOReD 2007. 5th Student Conference on. IEEE, 2007.

[12] Zhang, Dengsheng, et al. "Content-based image retrieval using Gabor texture features." Institute of Electrical and Electronics Engineers (IEEE) Transactions Pattern Analysis and Machine Intelligence (PAMI) (2000): 13-15.

[13] https://en.wikipedia.org/wiki/Local_binary_patterns 
Zannatul Azme, Khadiza Sultana Happy, Mohammad Farhad Bulbul; Flower Image Retrieval Using LBP, Wavelet Moments, Gabor Wavelet Features and Multiple Distance Functions. Advances in Image and Video Processing, Volume 6 No 5, October (2018); pp: 1-12

[14] Ojala, T., Pietikainen, M., Maenpaa, T.: Multiresolution gray-scale and rotation invariant texture Classification with local binary patterns. Institute of Electrical and Electronics Engineers (IEEE) Trans. Pattern Anal. Mach. Intell. 24 (7), 971-987 (2002)

[15] Bulbul, Mohammad Farhad, Yunsheng Jiang, and Jinwen Ma. "Real-Time Human Action Recognition Using DMMs-Based LBP and EOH Features." International Conference on Intelligent Computing. Springer, Cham, 2015.

[16] T.Prathiba, G. Soniah Darathi, "An efficient content based image Retrieval using local tetra pattern," International Journal of Advanced Research in Electrical, Electronics and Instrumentation Engineering, Vol. 2, Issue 10, October 2013.

[17] https://lyfat.wordpress.com/2012/05/22/euclidean-vs-chebyshev-vs-manhattan-distance/

[18] https://en.wikipedia.org/wiki/Cosine similarity

[19] http://people.revoledu.com/kardi/tutorial/Similarity/Correlation.html

[20] https://docs.tibco.com/pub/spotfire/7.0.0/doc/html/hc/hc city block distance.htm

[21] https://en.wikipedia.org/wiki/Minkowski_distance 